\title{
Non-alcoholic fatty liver disease and insulin resistance: importance of risk factors and histological spectrum
} Ana Cristina Guidorizzi de Siqueira ${ }^{a}$, Helma P. Cotrim ${ }^{a}$, Raquel Rocha ${ }^{a}$, Fernando M. Carvalho ${ }^{a}$, Luiz A.R. de Freitas ${ }^{a, b}$, Danyella Barreto ${ }^{a}$, Leandro Gouveia ${ }^{\mathrm{a}}$ and Luciana Landeiro ${ }^{\mathrm{a}}$

\begin{abstract}
Background Non-alcoholic fatty liver disease (NAFLD) has been associated with several metabolic conditions (MC) and secondary causes, but the relationship between insulin resistance (IR) and the underlying aetiology of NAFLD has not been extensively explored.
\end{abstract}

Objective To determine the frequency of IR among NAFLD patients and to describe IR according to risk factors and histological findings of the disease.

Methodology A case-series study of 64 patients with clinical and histological diagnosis of NAFLD. IR was calculated by homeostasis model assessment (HOMA) and IR was considered when HOMA $\geqslant 3$. Histological grades of NAFLD were: stage 1, steatosis isolated; stage 2, steatosis and inflammation; stage 3, steatosis and ballooning degeneration; stage 4, steatosis and fibrosis and/or Mallory bodies. Fibrosis was graded 0-4 (cirrhosis).

Results IR was found in $21(33 \%)$ patients. Among those with IR, 16 patients $(76 \%)$ had associated MC and five patients $(24 \%)$ had exposure to petrochemicals. The mean value of HOMA varied from 3.5 in NAFLD associated with MC to 1.6 in patients with exposure to

\section{Introduction}

Non-alcoholic fatty liver disease (NAFLD) is a clinicalpathological condition with growing prevalence in the modern world, characterized by a broad spectrum of liver damage, ranging from isolated steatosis to steatohepatitis $(\mathrm{NASH})$ and cirrhosis, in patients without history of alcohol abuse [1]. Although fatty liver has been considered an incidental pathological finding with a benign clinical course, more recent studies have shown that patients with NASH may progress to advanced liver fibrosis and it is now accepted that NASH is probably related to many cases of cryptogenic cirrhosis [2]. Approximately one-half of the patients with NASH develop liver fibrosis, $20 \%$ develop cirrhosis and $3 \%$ may progress to end-stage liver failure or liver transplantation $[3,4]$.

The pathogenesis of NAFLD is still not completely understood, but it has a strong association with insulin resistance (IR) and features of the metabolic syndrome $[5,6]$. NAFLD petrochemicals $(P<0.03)$. Waist circumference was the metabolic factor most strongly associated with IR $(P<0.005)$. Steatohepatitis (NASH) was observed in 54 $(84.3 \%)$ cases. The HOMA mean value was significantly higher in patients with advanced fibrosis.

Conclusions IR occurred in $33 \%$ of the NAFLD patients, being more frequent among those with $M C$ than among those with exposure to petrochemicals. The presence of IR in cases with advanced fibrosis suggests that it may influence the prognosis of NAFLD. Eur J Gastroenterol Hepatol 17:837-841 (c) 2005 Lippincott Williams \& Wilkins.

European Journal of Gastroenterology \& Hepatology 2005, 17:837-841

Keywords: non-alcoholic fatty liver disease, non-alcoholic steatohepatitis, insulin resistance

${ }^{a}$ Curso de Pós-graduação em Medicina e Saúde, Faculdade de Medicina da Universidade Federal da Bahia, Brazil and ' ${ }^{\mathrm{F}}$ undação Gonçalo Muniz, Fiocruz, Bahia, Brazil.

Correspondence and requests for reprints to Ana Cristina Guidorizzi de Siqueira, Rua Rio de Janeiro 591/702, Pituba, Salvador CEP 41830-401, Bahia, Brazil. Tel: +5571 3347 1055; fax: + 55712032741 ;

e-mail: anaguido@terra.com.br

Received 12 January 2005 Accepted 7 April 2005

has been suggested as one more component of this syndrome and the central role of IR and hyperinsulinaemia in its pathogenesis has been discussed [7,8].

Marchesini et al., using the homeostasis model assessment (HOMA) method, showed that IR was closely associated with the presence of NAFLD in a large series of patients, irrespective of body mass index (BMI), steatosis distribution or glucose tolerance [9].

This study aimed to determine the frequency of IR among NAFLD patients, and to describe IR according to some risk factors and histological findings of the disease.

\section{Methodology Patients}

From January 2002 to March 2004, 64 patients with clinical and histological diagnosis of NAFLD were selected for the present study. Most of them were 
referred to the hepatology unit of University Hospital at the Federal University of Bahia for ultrasound investigation of steatosis or elevated aminotransferases levels. Diabetic patients on insulin treatment were excluded.

Criteria of NAFLD/NASH were: alcohol consumption $<20 \mathrm{~g}$ /day; exclusion of $\mathrm{B}$ and $\mathrm{C}$ viral markers, autoantibodies and other serum markers for metabolic liver diseases; and histological diagnosis of NAFLD/NASH [1].

The study had the approval of the ethics committee of the Federal University of Bahia. All patients gave informed consent for participation.

\section{Clinical evaluation}

All patients were interviewed about alcohol intake and the presence of risk factors for NAFLD: diabetes, dyslipidaemia, drug use, abdominal surgery and exposure to chemicals. A complete physical examination included anthropometric data (height, weight, waist circumference) and blood pressure measures. The BMI was calculated by the Quetelet index: weight $(\mathrm{kg})$ divided by height ${ }^{2}(\mathrm{~m})$. Obesity was defined as BMI $\geqslant 30 \mathrm{~kg} / \mathrm{m}^{2}$ and overweight as BMI $\geqslant 25$ and $\leqslant 29.9 \mathrm{~kg} / \mathrm{m}^{2}$. The waist circumference, obtained in 56 patients, was measured at the midpoint between the lower border of the rib cage and the iliac crest.

The diagnosis of diabetes was made by the patient history or based on the criteria of the American Diabetes Association [10]. The metabolic syndrome was defined according to ATP III, as the presence of three or more of the following criteria: fasting glucose $\geqslant 110 \mathrm{mg} / \mathrm{dl}$; central obesity (waist circumference) $>102 \mathrm{~cm} \mathrm{(men)} \mathrm{and}$ $>88 \mathrm{~cm}$ (women); arterial pressure $\geqslant 130 / 85 \mathrm{mmHg}$ or pharmacologically treated; triglyceride levels $>150 \mathrm{mg} / \mathrm{dl}$ or current use of fibrates; high-density lipoprotein (HDL)-cholesterol $<40 \mathrm{mg} / \mathrm{dl} \quad$ (men) and $<50 \mathrm{mg} / \mathrm{dl}$ (women) [11].

Laboratory investigation was performed in all patients and included: fasting glucose, serum insulin, cholesterol, triglycerides, alanine aminotransferase (ALT), aspartate aminotransferase (AST), $\gamma$-glutamyltranspeptidase (GGT), alkaline phosphatase, total bilirubin, albumin, prothrombin time and iron status (serum iron, ferritin and transferrin saturation).

\section{Evaluation of insulin resistance}

Insulin was measured by an immunoenzymometric assay (IMMULITE 2000; DPC, Los Angeles, California, USA), with a medium value of $9.3 \mu \mathrm{U} / \mathrm{ml}$ and with intra-assay and inter-assay coefficients of variation for the quality control of $\pm 2.6 \%$. Serum glucose was measured by the glucose-oxidase method.
IR was calculated by means of HOMA: [serum insulin $(\mu \mathrm{U} / \mathrm{ml}) \times$ fasting glucose $(\mathrm{mmol} / \mathrm{l}) / 22.5]$. The HOMA method correlates closely with quantitative, functional techniques, such as the euglycaemic clamp method, and can be used for large surveys [12].

The use of HOMA in this study was validated by its correlation with another method to estimate insulin sensitivity, the Quantitative Insulin Check Index. Control HOMA values were also obtained from another study of the authors with 64 healthy volunteers, with age $>18$ years, non-obese, non-diabetic, without dyslipidaemia, with normal ALT levels and absence of serum markers of hepatitis B and hepatitis C. Among this group, the HOMA mean value was $1.48 \pm 0.9$. Patients were categorized as insulin resistant if the HOMA value was greater than 3.0.

\section{Histological evaluation}

Patients with ALT levels greater than 1.5 times the upper normal values for 3 months or more were selected for liver biopsy. Most of the specimens were obtained by the percutaneous suction needle biopsy technique and the typical sample sizes varied from 1 to $2 \mathrm{~cm}$. The specimens were stained with haematoxylin-eosin, periodic-acid Schiff, sirius red and Perls' Prussian blue. Histological classification was according to Matteoni et al. [13]: type 1, simple steatosis; type 2, steatosis with lobular inflammation; type 3 , type $2+$ ballooned hepatocytes; type 4 , type $3+$ the presence of either Mallory's hyaline or fibrosis. Types 3 and 4 were considered to be steatohepatitis. Fibrosis was classified into four stages according to Brunt et al. [14]: stage 0, without any fibrosis; stage 1, fibrosis limited to the perivenular or perisinusoidal area in zone 3; stage 2, perivenular and perisinusoidal fibrosis with portal fibrosis; stage 3, bridging fibrosis; stage 4, cirrhosis with or without residual perisinusoidal fibrosis. Perls' method was used to investigate iron overload.

\section{Statistical analysis}

All data were analysed using the Statistical Package for the Social Science program, version 9.0 [15]. Continuous variables in the text and in the tables are reported as the mean \pm standard deviation and categorical variables as frequencies and percentages. Student's $t$-test for unpaired data was used to compare groups when variables were normally distributed; otherwise the Mann-Whitney test was used. The $\chi^{2}$ or Fisher exact tests were used to compare categorical variables. Analysis of variance or the Kruskal-Wallis test was used for comparisons among several groups. Differences between groups were considered statistically significant at $P<0.05$.

\section{Results}

Among the 64 patients with clinical and histological diagnosis of NAFLD, 50 (78\%) were men and the mean 
age was $45.0 \pm 9.2$ years. All patients were asymptomatic and none of them had clinical evidence of liver failure.

The serum ALT and AST levels were elevated in 38 patients (59\%) and in 25 patients (39\%), respectively. The GGT levels were elevated in 20 patients $(31.0 \%)$. AST/ALT greater than 1 was observed in 14 patients (22\%) and, among them, only three patients (28\%) had advanced fibrosis or cirrhosis.

Table 1 presents the clinical, anthropometric and laboratory data in the patients, according to the presence of IR. The HOMA mean value was $2.7 \pm 1.7$, significantly higher than in controls. IR, defined as HOMA $\geqslant 3$, was present in 21 patients $(33 \%)$. There was no significant difference between groups according to gender; however, the mean age was significantly higher in the group with IR $(49.0 \pm 9.5$ versus $43.5 \pm 8.6$ years; $P<0.03)$. Among patients with NAFLD associated with exposure to chemicals, only five $(24 \%)$ had IR $(P<0.006)$. Most patients were overweight $\left(\mathrm{BMI} \geqslant 25\right.$ and $\left.\leqslant 29.9 \mathrm{~kg} / \mathrm{m}^{2}\right)$ and the BMI showed a significant correlation with IR $\left(30.0 \pm 3.9\right.$ versus $\left.27.6 \pm 3.0 \mathrm{~kg} / \mathrm{m}^{2} ; P<0.02\right)$. IR $(\mathrm{HOMA} \geqslant 3)$ was demonstrated in one of 10 normal weight subjects (10\%), in 12 of $38(32 \%)$ overweight cases and in eight of $16(50 \%)$ obese patients. Data also showed that IR was higher among patients with steatosis mainly distributed in the visceral area. A large waist circumference was present in 12 patients $(70.6 \%)$ with IR and in only six patients $(15.4 \%)$ without IR $(P<0.001)$. The other parameters, such as arterial pressure, serum cholesterol, triglyceride, aminotransferases, GGT levels and iron status, did not differ significantly between the two groups.

Table 2 shows that 32 patients (50\%) had exclusively metabolic risk factors, $24(37.5 \%)$ had exposure to chemicals associated with metabolic conditions and six patients $(9.4 \%)$ had exposure to chemicals as the only risk factor. One patient with NAFLD had a history of drug use (tamoxifen) and one was considered as cryptogenic. IR was significantly higher among patients with NAFLD exclusively associated with metabolic conditions than among patients with exposure to chemicals $(3.5 \pm 2.0$ versus $1.6 \pm 0.9 ; P<0.03)$.

The prevalence of positive criteria for the metabolic syndrome, according to the ATP III, is presented in Table 3 . Hypertriglyceridaemia $(>150 \mathrm{mg} / \mathrm{dl}$ ) was present in 33 patients (52\%), low HDL-cholesterol levels $(<40 \mathrm{mg} / \mathrm{dl}$ in men and $<50 \mathrm{mg} / \mathrm{dl}$ in women) in 30 patients $(47 \%)$,

Table 2 Homeostasis model assessment (HOMA) mean values according to risk factors in 64 patients with non-alcoholic fatty liver disease

\begin{tabular}{lcc}
\hline Risk factors & $n(\%)$ & $\begin{array}{c}\text { HOMA (mean } \pm \text { standard } \\
\text { deviation) }\end{array}$ \\
\hline Metabolic factors $^{\mathrm{a}}$ & $32(50)$ & $3.5 \pm 2.0$ \\
Exposure and metabolic factors $^{\mathrm{b}}$ & $24(37.5)$ & $2.0 \pm 1.0$ \\
Exposure to chemicals $^{\mathrm{c}}$ & $6(9.4)$ & $1.6 \pm 0.9$ \\
\hline
\end{tabular}

${ }^{\mathrm{a}, \mathrm{b}} P<0.001$

${ }^{\mathrm{b}, \mathrm{c}} P<0.4$

${ }^{\mathrm{a}, \mathrm{c}} P<0.03$

Table 1 Clinical, laboratory and anthropometric data in 64 patients with non-alcoholic fatty liver disease according to insulin resistance (IR)

\begin{tabular}{|c|c|c|c|c|}
\hline & Total $(n=64)$ & Patients with IR $(n=21)$ & Patients without IR $(n=43)$ & $P<$ \\
\hline Gender (male) (\%) & $50(78 \%)$ & $14(67 \%)$ & $36(84 \%)$ & 0.12 \\
\hline Age (years) & $45.0 \pm 9.2$ & $49.0 \pm 9.5$ & $43.5 \pm 8.6$ & 0.03 \\
\hline Exposure to chemicals & $30(47 \%)$ & $5(24 \%)$ & $25(58 \%)$ & 0.006 \\
\hline Without exposure to petrochemicals & $34(53 \%)$ & $16(76 \%)$ & $18(42 \%)$ & 0.006 \\
\hline Body mass index $\left(\mathrm{kg} / \mathrm{m}^{2}\right)$ & $28.0 \pm 3.5$ & $30.0 \pm 3.9$ & $27.6 \pm 3.1$ & 0.02 \\
\hline Waist circumference $(\mathrm{cm})^{\mathrm{a}}$ & $94.6 \pm 7.7$ & $99.4 \pm 7.4$ & $92.5 \pm 7.0$ & 0.001 \\
\hline $\begin{array}{l}\text { Waist circumference }>102 \mathrm{~cm} \text { (men) and } \\
>88 \mathrm{~cm} \text { (women) }^{\mathrm{a}}\end{array}$ & $18(32.0 \%)$ & $12(70.6 \%)$ & $6(15.4 \%)$ & 0.001 \\
\hline Systolic pressure $(\mathrm{mmHg})$ & $131.6 \pm 17.7$ & $134.5 \pm 21.4$ & $130.0 \pm 15.5$ & 0.36 \\
\hline Diastolic pressure $(\mathrm{mmHg})$ & $87.0 \pm 12.6$ & $87.0 \pm 15.6$ & $87.0 \pm 11.0$ & 0.75 \\
\hline Treatment for hypertension (\%) & $17(26.6 \%)$ & $6(28.6 \%)$ & $11(25.6 \%)$ & 0.80 \\
\hline Treatment for dyslipidaemia (\%) & $7(11.0 \%)$ & $3(14.0 \%)$ & $4(9.3 \%)$ & 0.67 \\
\hline Diabetes (\%) & $7(11.0 \%)$ & $4(19.0 \%)$ & $3(7.0 \%)$ & 0.20 \\
\hline Total cholesterol (mg/dl) & $211.0 \pm 59.0$ & $209.6 \pm 54.6$ & $212.0 \pm 6.0$ & 0.87 \\
\hline High-density lipoprotein-cholesterol (mg/dl) & $44.0 \pm 11.0$ & $42.0 \pm 1.0$ & $45.0 \pm 11.0$ & 0.29 \\
\hline Triglycerides (mg/dl) & $173.0 \pm 87.0$ & $181.0 \pm 91.0$ & $169.0 \pm 85.6$ & 0.50 \\
\hline AST (U/I) & $46.0 \pm 28.7$ & $56.7 \pm 31.0$ & $41.5 \pm 26.5$ & 0.05 \\
\hline ALT $(U / I)$ & $78.0 \pm 57.0$ & $90.0 \pm 75.0$ & $72.0 \pm 46.0$ & 0.24 \\
\hline AST/ALT (U//) & $0.8 \pm 0.6$ & $0.8 \pm 0.5$ & $0.7 \pm 0.6$ & 0.63 \\
\hline GGT (U/I) & $100.0 \pm 126.0$ & $117.0 \pm 157.0$ & $92.0 \pm 109.0$ & 0.85 \\
\hline Ferritin (ng/ml) & $385.0 \pm 269.6$ & $363.0 \pm 261.6$ & $396.0 \pm 276.0$ & 0.62 \\
\hline Transferrin saturation (\%) & $32.4 \pm 12.0$ & $35.5 \pm 15.0$ & $30.3 \pm 9.0$ & 0.13 \\
\hline Fasting glucose (mg/dl) & $104.0 \pm 24.0$ & $113.0 \pm 20.6$ & $99.5 \pm 24.6$ & 0.03 \\
\hline Serum insulin $(\mu \mathrm{U} / \mathrm{ml})$ & $10.3 \pm 5.6$ & $17.0 \pm 4.5$ & $7.3 \pm 2.4$ & 0.001 \\
\hline HOMA-IR & $2.7 \pm 1.7$ & $4.7 \pm 1.5$ & $1.8 \pm 0.6$ & 0.001 \\
\hline
\end{tabular}

Data are mean \pm standard deviation or number of cases and prevalence. AST, aspartate aminotransferase; ALT, alanine aminotransferase; GGT, $\gamma$-glutamyltranspeptidase; HOMA, homeostasis model assessment.

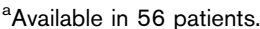


Table 3 Prevalence of positive criteria for the metabolic syndrome (MS) in 64 patients with non-alcoholic fatty liver disease

\begin{tabular}{ll}
\hline Criteria for MS & $n(\%)$ \\
\hline $\begin{array}{l}\text { Waist circumference }>102 \mathrm{~cm} \text { (men) and }>88 \mathrm{~cm} \\
\quad \text { (women) }^{\mathrm{a}}\end{array}$ & $18(32.0 \%)$ \\
Fasting glucose $\geqslant 110 \mathrm{mg} / \mathrm{dl}$ & $17(26.6 \%)$ \\
$\begin{array}{l}\text { High-density lipoprotein-cholesterol }<40 \mathrm{mg} / \mathrm{dl} \text { (men) } \\
\quad \text { and }<50 \mathrm{mg} / \mathrm{dl} \text { (women) }\end{array}$ & $30(47.0 \%)$ \\
$\begin{array}{l}\text { Triglycerides }>150 \mathrm{mg} / \mathrm{dl} \text { or treatment with fibrates } \\
\text { Arterial pressure } \geqslant 130 \times 85 \mathrm{mmHg} \text { or pharmacologically }\end{array}$ & $33(51.6 \%)$ \\
$\quad$ treated & $26(44.0 \%)$ \\
\hline
\end{tabular}

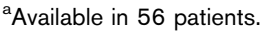

hypertension (blood pressure $\geqslant 130 / 85 \mathrm{mmHg}$ ) in 26 patients $(44 \%)$, central distribution of obesity (waist circumference $>102 \mathrm{~cm}$ in men and $>88 \mathrm{~cm}$ in women) in 18 patients $(32 \%)$ and hyperglycaemia in 17 patients $(27 \%)$.

The metabolic syndrome was observed in 19 cases (29.7\%) of NAFLD (three or more criteria). Among patients with the metabolic syndrome, $13(68 \%)$ had NAFLD associated with metabolic conditions and six (32\%) had NAFLD associated with exposure to chemicals. The HOMA mean value was significantly higher in patients with metabolic syndrome $(3.6 \pm 2.1$ versus $2.4 \pm 1.4 ; P<0.003)$.

Among other metabolic risk factors for NAFLD, waist circumference showed the strongest association with IR (Table 4).

The histological findings in liver biopsies of 64 patients were: three $(4.7 \%)$ had simple steatosis, seven (11\%) had steatosis and lobular inflammation, four (6.3\%) had steatosis and ballooned hepatocytes, and 50 (78\%) had NASH with fibrosis. Fifty-four patients $(84.3 \%)$ were classified as NASH (types 3 and 4). Table 5 describes the grades of fibrosis in liver biopsies and their correlation with HOMA mean values. Mild or grade 1 fibrosis was the most frequent and the HOMA mean value was significantly higher in patients with more advanced fibrosis (stages 3 and 4), compared with patients without fibrosis $(5.2 \pm 2.0$ versus $1.9 \pm 0.9 ; P<0.001)$ and with mild fibrosis $(5.2 \pm 2.0$ versus $2.6 \pm 1.5 ; P<0.002)$.

Iron overload, analysed by Perls' method, was present in 20 patients $(31 \%)$. The HOMA mean values were $2.6 \pm 1.3$ in patients with NAFLD and iron overload, and $2.8 \pm 1.9$ in patients without iron overload (not significant).

\section{Discussion}

The frequency of IR among patients with NAFLD in our series (33\%) was much lower than in previous reports by other authors [7,9]. This difference may be justified by the great variation between the values of HOMA-R considered as IR and by the fact that these previous
Table 4 Homeostasis model assessment (HOMA) mean values according to metabolic factors in 64 patients with non-alcoholic fatty liver disease

\begin{tabular}{lcccc}
\hline Metabolic factors & & $n$ & $\begin{array}{c}\text { HOMA (mean } \pm \\
\text { standard deviation) }\end{array}$ & $P<$ \\
\hline Body mass index class & $\begin{array}{c}\text { Normal } \\
\text { weight } \\
\text { Overweight }\end{array}$ & 10 & $1.9 \pm 1.1$ & 0.08 \\
& Obesity & 15 & $39.2 \pm 1.4$ & \\
Waist circumference ${ }^{a}$ & Normal & 34 & $2.0 \pm 1.4$ & 0.005 \\
& Elevated & 15 & $3.8 \pm 2.3$ & \\
Dyslipidaemia & Yes & 43 & $2.9 \pm 1.8$ & 0.32 \\
Diabetes & No & 21 & $2.4 \pm 1.5$ & 0.02 \\
& Yes & 7 & $4.4 \pm 2.2$ & 0.02 \\
Hypertension & No & 57 & $2.5 \pm 1.5$ & \\
& Yes & 22 & $3.0 \pm 2.2$ & 0.50 \\
& No & 42 & $2.6 \pm 1.4$ &
\end{tabular}

${ }^{\mathrm{a}}$ Available in 56 patients.

Table 5 Homeostasis model assessment (HOMA) mean values according to fibrosis grades in 64 patients with non-alcoholic fatty liver disease

\begin{tabular}{lrc}
\hline Histology & $n(\%)$ & $\begin{array}{c}\text { HOMA (mean } \pm \text { standard } \\
\text { deviation) }\end{array}$ \\
\hline Without fibrosis $^{\mathrm{a}}$ & $13(20.0 \%)$ & $1.9 \pm 0.9$ \\
Mild fibrosis (grades 1 and 2) $^{\mathrm{b}}$ & $44(69.0 \%)$ & $2.6 \pm 1.5$ \\
Advanced fibrosis (grades 3 and 4) $^{\mathrm{c}}$ & $7(11.0 \%)$ & $5.2 \pm 2.0$
\end{tabular}

${ }^{\mathrm{a}, \mathrm{b}} P<0.3$;

b,c $P<0.002$

${ }^{\mathrm{a}, \mathrm{c}} P<0.001$.

studies included mainly obese individuals and the present study evaluated subjects with several risk factors for NAFLD, including metabolic and secondary factors.

The analysis of IR according to risk factors showed a remarkable difference of HOMA values among patients with NAFLD associated with metabolic conditions (obesity, diabetes, dyslipidaemia), when compared with NAFLD related to exposure to chemicals. The higher occurrence of IR in cases of NAFLD associated with metabolic conditions confirms previous data [7-9]. NAFLD related to exposure to petrochemical products has been observed in such workers in Brazil. The absence of IR in the majority of these patients suggests that exposure may be an independent risk factor for the development of this liver disease and that other mechanisms may be involved in its pathogenesis [16,17].

IR was strongly associated with the metabolic syndrome in patients with NAFLD related to metabolic conditions when compared with exposure to chemicals. The most prevalent positive criteria for metabolic syndrome in our series was hypertriglyceridaemia, followed by low HDLcholesterol levels and hypertension. However, there was no significant association of IR with the presence of either dyslipidaemia or hypertension.

HOMA values differed significantly between obese and lean subjects. These data support the role of adipose tissue in the development of IR. Recent studies have 
demonstrated that the risk of NAFLD seems to be particularly associated with central obesity rather than peripheral obesity [7]. It is known that visceral adipocytes are more prone to lipolysis than those from the periphery and the free fatty acids released from central adipose sites drain directly to the liver via the portal vein. In our series, IR was much more strongly associated with central obesity than with BMI. This finding suggests that waist circumference may be a better parameter for identifying the metabolic syndrome among NAFLD patients.

Steatohepatitis with fibrosis was the most frequent finding. These data probably reflect the criteria for selecting cases for liver biopsy. Only patients with persistent high levels of aminotransferases were selected and probably those with more advanced liver disease were included.

Risk factors have been identified as predictors for the development of progressive fibrosis and cirrhosis in patients with NAFLD. Obesity and diabetes are considered the strongest predictors for fibrosis [18]. Additional risk factors include older age, high ALT levels, AST/ALT ratio $>1$, hypertension and hypertriglyceridaemia [19]. Although IR has an important role in the development of hepatic steatosis, the association of IR with progression of fatty liver to advanced fibrosis is still not defined. The higher IR index in patients with advanced liver disease could be the result of the usual association between hyperinsulinaemia and liver failure. Patients with advanced fibrosis or cirrhosis are usually insulin resistant and the hyperinsulinaemia in these cases is the consequence of impaired insulin degradation [20]. However, it is not usually associated with milder forms of liver disease. In our series, all patients had normal liver function and cirrhosis was present in only three cases (4.8\%). According to fibrosis grades, the HOMA mean value was significantly higher in those patients with more advanced fibrosis (grades 3 and 4). The association of IR with advanced fibrosis in this study suggests that IR may be probably a predictor for progression of the disease and not only a consequence.

In conclusion, this study among NAFLD patients suggests that IR is much more strongly associated with metabolic conditions than with exposure to petrochemicals. The association of IR and steatohepatitis with fibrosis suggests that it may have influence in the prognosis of NAFLD.

\section{Conflict of interest}

None declared.

\section{Authors' contributions}

A.C.G.S. is the main author and H.P.C. is the second author. R.R. is the dietician that helped us to obtain the anthropometric data. F.M.C. is the epidemiologist that contributed to the statistical analysis. L.A.R.F. is a pathologist and was mainly responsible for the histological evaluation of biopsy specimens. D.B., L.G. and L.L. are graduate students that helped evaluate the patients and obtain the results.

\section{References}

1 Brunt EM. Nonalcoholic steatohepatitis. Definition and pathology. J Liver Dis 2001; 21:3-14

2 Caldwell S, Oelsner D, lezzoni J, Hespenheide E, Battle E, Driscoll C. Cryptogenic cirrhosis. Clinical characterization and risk factors for underlying disease. Hepatology 1999; 29:664-669.

3 Sheth SG, Gordon FD, Chopra S. Nonalcoholic steatohepatitis. Ann Intern Med 1997; 126:137-145.

4 Falck-Ytter Y, Younossi Z, Marchesini G, McCullough AJ. Clinical features and natural history of nonalcoholic steatosis syndromes. Seminars Liver Dis 2001; 21:17-26.

5 Marchesini G, Bugianesi E, Forlani G, Cerreli F, Lenzi M, Manini R, et al. Nonalcoholic fatty liver, steatohepatitis and the metabolic syndrome. Hepatology 2003; 37:917-923.

6 Marchesini G, Brizi M, Bianchi G, Tomassetti S, Bugianesi E, Lenzi M, et al. Non alcoholic fatty liver disease. A feature of the metabolic syndrome. Diabetes 2001; 50:1844-1850.

7 Chitturi S, Abeygunasekera S, Farrell G, Holmes-Walker J, Hui J, Fung C, et al. NASH and insulin resistance: insulin hypersecretion and specific association with the insulin resistance syndrome. Hepatology 2002; 35:373-378.

8 Pagano G, Pacini G, Musso G, Gambino R, Mecca F, Depetris N, et al. Nonalcoholic steatohepatitis, insulin resistance and metabolic syndrome: further evidence for an etiologic association. Hepatology 2002; 35 : 367-371.

9 Marchesini G, Bizi M, Morselli-Labate AM, Bianchi G, Bugianesi E, McCullough AJ, et al. Association of nonalcoholic fatty liver disease with insulin resistance. Am J Med 1999; 107:450-455.

10 Report of the Expert Committee on the Diagnosis and Classification of Diabetes Mellitus. Diabetes Care 1997; 20:1183-1195.

11 Executive Summary of the Third Report of the National Cholesterol Education Program (NCEP) Expert Panel on Detection, Evaluation and Treatment of High Blood Cholesterol in Adults (Adult Treatment Panel III). J Am Med Assoc 2001; 285:2486-2497.

12 Matthews DR, Hosker JP, Rudenski AS, Naylor BA, Treacher DF, Turner RC. Homeostasis model assessment: insulin resistance and $\beta$-cell function from fasting plasma glucose and insulin concentrations in man. Diabetologia 1985; 28:412-419.

13 Matteoni C, Younossi Z, Gramlich T, Boparai N, Liu YC, McCullough AJ. Nonalcoholic fatty liver disease: a spectrum of clinical and pathological severity. Gastroenterology 1999; 116:1413-1419.

14 Brunt EM, Janney CG, Di Bisceglie AM, Brent A, Neuschwander-Tetri BA, Bacon B. Nonalcoholic steatohepatitis: a proposal for grading and staging the histological lesions. Am J Gastroenterol 1999; 94:2467-2474.

15 Norussis MJ. Statistical Package for the Social Sciences (SPSS), version 9.0. Chicago, IL: SPSS Inc.; 1998.

16 Cotrim H, Andrade Z, Parana R, Portugal M, Lyra L, Freitas L. Nonalcoholic steatohepatitis: a toxic liver disease in industrial workers. Liver 1999; 19:299-304.

17 Cotrim H, Freitas LAR, Freitas C, Braga L, Sousa R, Carvalho F, et al. Clinical and histopathological features of NASH in workers exposed to chemical with or without associated metabolic conditions. Liver Int 2004; 24:131-135.

18 Ratziu V, Giral P, Charlotte F, Bruckert E, Thibault V, Theodorou I, et al. Liver fibrosis in overweight patients. Gastroenterology 2000; 118:1117-1123.

19 Angulo P, Keach JC, Batts KP, Lindor KD. Independent predictors of liver fibrosis in patients with nonalcoholic steatohepatitis. Hepatology 1999; 30:1356-1362.

20 Petrides AS, Vogt C, Schulze-Berge D, Matthews D, Strohmeyer G. Pathogenesis of glucose intolerance and diabetes mellitus in cirrhosis. Hepatology 1994; 19:616-627. 